\title{
Balsam Peru Oil
}

National Cancer Institute

\section{Source}

National Cancer Institute. Balsam Peru Oil. NCI Thesaurus. Code C107269.

The essentail oil of Myroxylon balsamum. Balsam peru oil is used primarily in parfumery and skin care preparations. 